\title{
Effect of Memantine on Oxidative and Antioxidant Indexes Among Elderly Patients with Prediabetes and Mild Cognitive Impairment
}

Raheleh Alimoradzadeh*, Hossein Mirmiranpour*, Payam Hashemi, Shaghayegh Pezeshki and Salome Sadat Salehi*

Endocrinology and Metabolism Research Center (EMRC), Vali-Asr Hospital, School of Medicine, Tehran University of Medical Sciences, Tehran, Iran

\begin{abstract}
Objective: Mild cognitive impairment $(\mathrm{MCl})$ is a cognitive syndrome in the elderly individuals. $\mathrm{MCl}$ drug treatment is still under debate. On the other hand, $\mathrm{MCl}$ progress is higher in people with diabetes mellitus type 2 . This study was designed to determine the effect of Memantine on oxidant and antioxidant indexes among the elderly with prediabetes and $\mathrm{MCl}$.
\end{abstract}

Method: The present study is a double-blind randomized clinical trial which was conducted on 50 elderly patients over 60 years of age who had been diagnosed with prediabetes and $\mathrm{MCl}$. Subjects were divided into intervention and control groups. The intervention group was treated with Memantine and Metformin and the control group received Metformin only. The data were collected before and after the study by blood tests and clinical examination and analyzed by using correlation test, independent and paired sample t-test in SPSS v.23 software.

Results: The subjects included 22 men and 28 women. The mean age subject was $72.87 \pm 3.95$. Regarding the control group, the oxidative indexes including Advanced Glycation End Products (AGEs) and Advanced oxidation protein products (AOPP) decreased significantly ( $p$ value $<0.001$, df: 48). Regarding the intervention group, the oxidant indexes including AGEs and AOPP significantly decreased. In addition, antioxidant indexes such as LAP and Lecithin-cholesterol acyltransferase (LCAT) indexes increased considerably ( $p$ value<0.001, df: 48 ).

Conclusion: Based on the results, the combination therapy with Memantine and Metformin for elderly patients with Prediabetic State and $\mathrm{MCl}$ may decrease oxidant indexes and increase the effect on antioxidant indices.

Keywords: Cognitive dysfunction; Prediabetic state; Oxidants; Antioxidants

\section{Introduction}

The elderly population of the world has grown rapidly over the last two centuries, due to factors such as declining birth rate, increased life expectancy, and medical advances [1]. By 2050, the world's elderly population is expected to reach more than two billion people [2]. With the increasing number of elderly people, the prevalence of age-related diseases has increased, among which Alzheimer is regarded as one of the most important of these diseases. Predictions suggest that the population of the elderly with Alzheimer's will reach to 115 million by 2050 [3].

Cognitive impairment is a neurological disorder in the elderly people which includes Mild Cognitive Impairment (MCI), Alzheimer's disease (AD), and Vascular Dementia (VD). AD is the most common cause of dementia among the elderly people and is affected by a cascade process including various mechanisms such as amyloid deposition, inflammation, high amounts free radicals, synapses and neurons loss and dysfunction of neurotransmitters [4]. MCI is a pre-stage of Alzheimer's disease and the transitional phase of the cognitive decline associated with age to the more serious cognitive decline in dementia $[5,6]$. The mild cognitive impairment includes two types of amnestic and non-amnestic, memory loss is the main symptom of the Amnestic $\mathrm{MCI}$, and it is the main non-amnestic symptom in other cognitive domains [7]. As compared to the elderly with Normal Cognitive Aging, the elderly with MCI are 3-5 times more likely to be exposed to AD [8]. Diabetes mellitus type 2 is a chaotic public health problem which is on the rise. In addition, the effects of diabetes on the peripheral nervous system were confirmed although its impact on higher mental functions has been neglected [9]. The prevalence of diabetes and cognitive impairment is accelerated in the elderly ages [10]. The incidence of MCI in people with diabetes is higher than those without diabetes, but its pathophysiology is unclear [11]. It has been argued that high levels of glucose can adversely affect cognition without revealing the symptoms of diabetes mellitus [12]. Prediabetic State is the period before the onset of diabetes and is a condition including Impaired Fasting Glucose (IFG) and Impaired Glucose Tolerance (IGT) [13]. Prediabetes and diabetes mellitus appear to increase oxidative stress by creating reactive oxygen species (ROS) [14].

High levels of glucose can lead to cognitive impairment through the mechanisms of Advanced Glycation End Products (AGEs), Oxidative stress, insulin resistance, and microvascular disease of the central nervous system [15,16]. Further, increased levels of glucose in the cell lead to nonenzymatic glycosylation of cellular proteins, lipoproteins, or nucleic acids [17]. On the other hand, AGEs increase over the elderly ages [18].

During 1993-2003, four inhibitors of Cholinesterase and Memantine have been approved for the symptomatic treatment of

*Corresponding author: Hossein Mirmiranpour, Endocrinology and Metabolism Research Center (EMRC), Vali-Asr Hospital, School of Medicine, Tehran University of Medical Sciences, Tehran, Iran, Tel: +9821-88417918, Fax: +9821-64432466 E-mail: h_mirmiranpoor@yahoo.com

Raheleh Alimoradzadeh, Firoozabadi Clinical Research Development unit (FACRDU), Iran University of Medical Sciences(IUMS), Tehran,Iran; E-mail: Alimoradzadeh.r@iums.ac.ir

Received January 08, 2019; Accepted February 12, 2019; Published February 18, 2019

Citation: Alimoradzadeh R, Mirmiranpour H, Hashemi P, Pezeshki S, Salehi SS (2019) Effect of Memantine on Oxidative and Antioxidant Indexes Among Elderly Patients with Prediabetes and Mild Cognitive Impairment. J Neurol Neurophysiol 10: 483. doi:10.4172/2155-9562.1000483

Copyright: () 2019 Alimoradzadeh R, et al. This is an open-access article distributed under the terms of the Creative Commons Attribution License, which permits unrestricted use, distribution, and reproduction in any medium, provided the original author and source are credited. 
Alzheimer's disease in the United States and Europe [19], but none has been confirmed for the treatment of individuals with $\mathrm{MCI}$ and require further research on the MCI therapeutic model [8]. Memantine is a non-competitive inhibitor for $\mathrm{N}$-methyl-D-aspartate receptor, which prevents over-stimulation of this NMDA-receptor by Glutamate (a defective memory factor in Alzheimer's disease) [20]. A large body of research suggested that Memantine can reduce the production of free radicals $[21,22]$.

So far, no systematic and comprehensive research has been conducted on the effect of Memantine on the oxidative and antioxidant indexes of patients with MCI and prediabetes although they were proposed in the form of a single study. In addition, considering the importance of oxidative stress among the studies conducted during the recent and the role of diabetes mellitus in intensifying and accelerating the progression of MCI, this study was conducted to determine the effect of Memantine on oxidative and antioxidant indexes among patients with prediabetes and MCI.

\section{Methodology}

This study is a randomized and double blind clinical trial study. The population included elderly patients with Prediabetes and MCI who referred to Memory and Behavioral Neurology Department located in Rouzbeh Hospital. Inclusion criteria were the ages between 65 and 80, comorbidity with Prediabetes and MCI. Exclusion criteria included the unwillingness to continue study, depression, history of chronic underlying diseases such as renal, pulmonary and liver diseases, history of treatment with memantine and other treatments for mild cognitive impairment, history of degenerative diseases like cancer or AIDS, alcohol consumption in the past month, ongoing smoking habit, present consumption of drugs and supplements and antioxidant vitamins, the occurrence of adverse effects of the drug or its lack of effect. Individuals were selected for physical and mental examination by a neurologist during an initial visit. The mild cognitive impairment was determined according to the criteria in Table 1 by the neurologist. Then, based on Fasting Blood Sugar test (between 100 and 126 mg /dL), patients with Prediabetic State were selected during two visits.

Patients who entered the memory and neurology clinic of Roozbeh Hospital were randomly assigned into intervention and control groups according to their eligibility for inclusion ( $\mathrm{N}=25$ for each group). The sample size based on the following formula and ( $\alpha, 1: 0, \beta: 25 / 25: d$, 7/28: S1, 15: S2) which were excluded in other studies, was estimated to be about 18 individuals

The sample size based on the following formula $(\alpha, 1: 0, \beta: 25 / 25: d$, 7/28: S1, 15: S2)

$$
n=\frac{\left(Z 1-\frac{\alpha}{2}+Z 1-\beta\right)^{2}\left(S_{1}^{2}+S_{2}^{2}\right)}{(d)^{2}}
$$

By a $20 \%$ drop which is equivalent to 4 individuals, as well as considering the referring statue of the patients to the clinic, the total number of subjects was 25 in each group (50 in total). In the Memantine hydrochloride intervention group, initially $5 \mathrm{mg}$ of Memantine was prescribed per day under the supervision of a neurologist, which gradually increased to $10 \mathrm{mg}$ per day regarding the neurologist's agreement. In addition, the patients in the intervention group were treated by $500 \mathrm{mg}$ Metformin tablets. Regarding the control group, only Metformin was given as $500 \mathrm{mg}$ tablets based on related neurologist. Based on the results, it was found that Memantine is not antagonist with Metformin [23-27].

Then, the subjects' blood pressure was measured by a standard barometer (Riester, Bing Ben adults, Germany) after 10 minutes of rest and while sitting. The mean of the two measurements within 5 minutes was recorded as systolic and diastolic blood pressures of the individuals. The subjects' weight subject was recorded by the Digital Scale (Beurer, GS49, Germany) wearing a light weight dress and the nearest $0.1 \mathrm{~kg}$. In addition, the height of the subjects was measured and recorded at the precision of $0.1 \mathrm{~cm}$ by a standard stadiometer, standing and without shoe. Body mass index (BMI) was calculated based on the Quetelet formula ((height in meter) 2/weight in kilograms). The waist circumference of the subjects subject was measured in the middle of the lower edge of the ribs and iliac crest, and the hip circumference was measured at the wings of the widest hips by a flexible meter. First, at least after 10-hour fasting, $10 \mathrm{ml}$ of venous blood sample was taken from each subject in the hospital environment. The blood samples of both groups were prepared and collected according to the principles of sterilization during the preadministration of Memantine and the samples were stored at $-80^{\circ} \mathrm{C}$.

Fasting blood glucose (FBD) levels are measured by glucose oxidase colorimetric assay and kit made by Pars Test company in Iran and by the BT-3000 Auto-Analyzer (plus) device made by Biotechnica factory in Italy. In order to evaluate the oxidant and antioxidant indexes, fluorometry was applied to determine the activity of lipoprotein lipase (LPL) in serum by LPL Activity Assay made by Roar Company in the USA and the activity of lecithin cholesterol acyl transferase (LCAT) was determined which was produced by Calbiochem Company in the USA. For this purpose, Floustar Spectrofluorometer device manufactured by BMG Company in Germany was used. The activity of serum Paraoxonase 1 - PON1 was assayed based on enzymatic colorimetric assay by Fully Automated Paraoxonase Activity Measurement Kit made by Rel Assay Diagnostics in Turkey and Sunrise ELISA micro plate reader made by Tecan Company in Austria. Advanced Oxidation Protein Products (AOPP) was determined by using Shimadzu Spectrophotometer, model UV-3100, manufactured by the Japan and based on the Kalousova method. In order to measure Ferric Reducing Ability of Plasma (FRAP), $750 \mu \mathrm{l}$ of FRAP reagent was added to $25 \mu \mathrm{l}$ of plasma in the test tubes, and their absorbance was read in $593 \mathrm{~nm}$ wave length.

In the next procedure, the FRAP reagent was prepared by mixing $300 \mathrm{mM}$ 3-acetate buffer with $\mathrm{pH} 3.6,10 \mathrm{mM}$ 2,4,6-tripyridynl-striazine (TPTZ) in $40 \mathrm{mM}$ chloride acid, as well as $20 \mathrm{Mm}$ hexahydrate ferric chloride, in proportions 1.1.10. In this method, the antioxidant agents found in the test sample resulted in reducing the ferric terpyridyl triazine $\left(\mathrm{TPTZ}^{-} \mathrm{Fe}^{3+}\right.$ ) complex to ferrous form (TPTZ- $\mathrm{Fe}^{2+}$ ), which is blue in acidic medium and has an optical absorption at $593 \mathrm{~nm}$

When there is a concern about the change in cognition, expressed by the patients, an aware person, or specialist

When there is objective memory impairment in memory, performance function, attention, language, or spatial visibility.

When the patient is independent in functional abilities although he may need help, or has mild disruptions in more complex tasks such as financial management, navigation in unfamiliar places, and so on.

When the patient has no criterion for dementia.

NIA/AA: National Institute on Aging and Alzheimer's Association

Table 1: Clinical diagnostic criteria for mild cognitive impairment based on NIA/AA criteria (23). 
Page 3 of 5

wavelength. The above reaction rate has a linear relationship with the reduction potential of the sample. Then, the advanced glycation end products (AGEs) was measure by diluting the serum using phosphate buffer (PBS) with $\mathrm{pH}$ 7.4: as much as 1 to 50. In addition, the intensity of the fluorescence was measured in $440 \mathrm{~nm}$ emission wavelength and the $350 \mathrm{~nm}$ exciting wavelength. Further, the fluorescence intensity was expressed in term of the fluorescence emission percentage. Choosing a treatment method was done by a colleague who was unaware of the treatment methods. The study was performed based on double blind method (from the results analyzer and investigator of experimental results). The intervention group was received a daily dose of $5 \mathrm{mg}$ of Memantine, which gradually increased to $10 \mathrm{mg}$ per day. The duration of Memantine administration was 6 months. It was ethically allowed to prescribe Memantine for subjects. The drug is one of the most widely accepted medicines for treating the patients with MCI.

The blood samples of both groups were prepared and collected after 6 months of re-administration of Memantine, following the sterilization principles and based on the conditions stated in the first stage of sampling (Figure 1).

The results were analyzed using SPSS v.23 software. Descriptive data were reported by using mean, standard deviation and frequency. Data were analyzed by independent $t$-test for comparing two groups of control and intervention, paired t-test was applied for comparing the results before and after studying each group, and Pearson correlation test was used to examine the relationships between some variables (Tables 2 and 3).

\begin{tabular}{|l|c|c|c|}
\hline & \multicolumn{2}{|c|}{ Group } & \multirow{2}{*}{ p-value } \\
\cline { 1 - 3 } & Control & Intervention & \\
\hline Age (Years) & $73.1 \pm 4.2$ & $72.6 \pm 3.7$ & 0.72 \\
\hline Weight $(\mathrm{kg})$ & $75.6 \pm 12.1$ & $80.2 \pm 10.1$ & 0.14 \\
\hline Body mass index $(\mathrm{kg} / \mathrm{m} 2)$ & $26.8 \pm 4.7$ & $28.9 \pm 3.1$ & 0.08 \\
\hline Waist circumference $(\mathrm{cm})$ & $97.6 \pm 8.6$ & $102.3 \pm 10.1$ & 0.08 \\
\hline Height $(\mathrm{m})$ & $1.6 \pm 0.1$ & $1.6 \pm 0.1$ & 0.36 \\
\hline
\end{tabular}

Table 2: Anthropometric features of the subjects.

\begin{tabular}{|l|l|c|c|c|}
\hline \multirow{2}{*}{\multicolumn{2}{|c|}{}} & \multicolumn{2}{c|}{ Group } & \multirow{2}{*}{ p-value } \\
\cline { 3 - 5 } & Before study & $75.6 \pm 12.2$ & $80.2 \pm 10.1$ & P: 0.15 \\
\hline Weight & After study & $75.6 \pm 11.9$ & $80.2 \pm 10.6$ & P: 0.16 \\
\hline & & $p$-value: 0.81 & p-value : 0.91 & \\
\hline BMI & Before study & $26.8 \pm 4.7$ & $28.9 \pm 3.1$ & P: 0.07 \\
\hline & After study & $26.8 \pm 4.5$ & $28.9 \pm 3.4$ & P: 0.76 \\
\hline & & $p$-value : 0.90 & p-value : 0.85 & \\
\hline Systolic Blood Pressure & Before study & $129.2 \pm 8.1$ & $123.20 \pm 9.8$ & P: 0.02 \\
\hline & After study & $122.6 \pm 7.1$ & $120.40 \pm 9.7$ & P: 0.05 \\
\hline & & $p$-value $<0.001$ & $p$-value : 0.005 & \\
\hline Diastolic Blood Pressure & Before study & $76 \pm 5.7$ & $76.8 \pm 4.7$ & P: 0.59 \\
\hline & After study & $74 \pm 5.7$ & $76 \pm 5.7$ & P: 0.23 \\
\hline & & $p$-value: 0.03 & $p$-value: 0.16 & \\
\hline
\end{tabular}

Table 3: Comparison of weight, body mass index, and blood pressure in both groups before and after intervention (Weight $(\mathrm{Kg})$, BMI $\left(\mathrm{kg} / \mathrm{m}^{2}\right)$, Systolic Blood Pressure $(\mathrm{mmHg})$, and Diastolic Blood Pressure $(\mathrm{mmHg}))$.

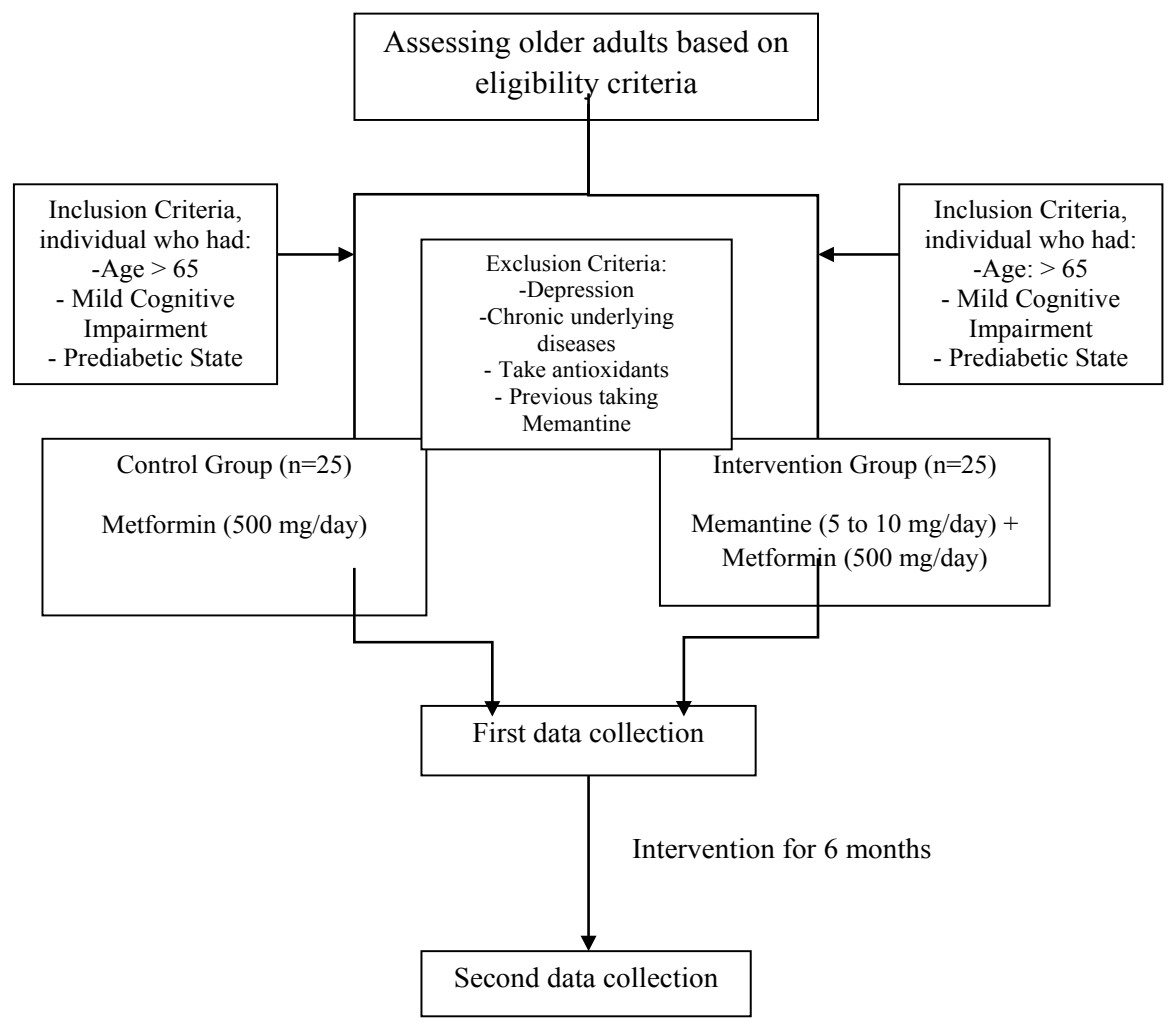

Figure 1: Flowchart of the subjects through the intervention. 


\begin{tabular}{|c|c|c|c|c|}
\hline & \multicolumn{2}{|c|}{ Group } & \multirow{2}{*}{$p$-value } \\
\hline & & Control & Intervention & \\
\hline \multirow{2}{*}{$\begin{array}{l}\text { Fasting Blood Sugar } \\
\text { (FBS) }\end{array}$} & Before study & $116.1 \pm 5.6$ & $117.2 \pm 5.9$ & P: 0.44 \\
\hline & After study & $105.7 \pm 3.97$ & $106.6 \pm 4.3$ & P: 0.47 \\
\hline & & $\mathrm{p}$ value $<0.001$ & $p$ value $<0.001$ & \\
\hline \multirow{2}{*}{$\begin{array}{l}\text { Advanced glycation } \\
\text { end-product (AGE) }\end{array}$} & Before study & $65.3 \pm 8.3$ & $70.7 \pm 4.7$ & P: 0.007 \\
\hline & After study & $63.5 \pm 8.4$ & $65.9 \pm 5.1$ & $P: 0.22$ \\
\hline & & $P<0.001$ & $P<0.001$ & \\
\hline \multirow{2}{*}{$\begin{array}{l}\text { Advanced oxidation } \\
\text { protein products } \\
\text { (AOPP) }\end{array}$} & Before study & $126.11 \pm 32.4$ & $136.8 \pm 18.8$ & $P: 0.16$ \\
\hline & After study & $124.18 \pm 32.4$ & $133.7 \pm 18.9$ & $P: 0.21$ \\
\hline & & $P$ value $<0.001$ & $P$ value $<0.001$ & \\
\hline \multirow{2}{*}{$\begin{array}{l}\text { Ferritin Reducing } \\
\text { Ability of Plasma } \\
\text { (FRAP) } \\
\end{array}$} & Before study & $1122.8 \pm 326.6$ & $1008.3 \pm 177.8$ & P: 0.13 \\
\hline & After study & $1216.2 \pm 269.3$ & $1104.5 \pm 157.6$ & $P: 0.08$ \\
\hline & & P: 0.30 & P: 0.09 & \\
\hline \multirow{3}{*}{$\begin{array}{l}\text { Paraoxonase-1 } \\
\text { (PON1) }\end{array}$} & Before study & $36.7 \pm 5.4$ & $32.2 \pm 9.1$ & $P: 0.06$ \\
\hline & After study & $38.9 \pm 8.8$ & $37.5 \pm 9.1$ & $P: 0.57$ \\
\hline & & $P: 0.26$ & P: 0.07 & \\
\hline \multirow{2}{*}{$\begin{array}{l}\text { Lipoprotein lipase } \\
\text { (LPL) }\end{array}$} & Before study & $26.95 \pm 1.4$ & $20.9 \pm 1.4$ & $P<0.001$ \\
\hline & After study & $28.07 \pm 2.9$ & $27.4 \pm 4.4$ & P: 0.58 \\
\hline & & P: 0.12 & $P<0.001$ & \\
\hline \multirow{2}{*}{$\begin{array}{l}\text { Lecithin-cholesterol } \\
\text { acyltransferase } \\
\text { (LCAT) }\end{array}$} & Before study & $36.37 \pm 1.1$ & $32.6 \pm 1.1$ & $P<0.001$ \\
\hline & After study & $38.26 \pm 3.9$ & $37.5 \pm 4.2$ & $P: 0.54$ \\
\hline & & $P: 0.06$ & $P<0.001$ & \\
\hline \multirow{2}{*}{$\begin{array}{l}\text { Haemoglobin subunit } \\
\text { alpha } 1 \text { (HBA1) }\end{array}$} & Before study & $6.1 \pm 0.5$ & $9.1 \pm 1.5$ & $P<0.001$ \\
\hline & After study & $6.1 \pm 0.4$ & $7.7 \pm 1.7$ & $P<0.001$ \\
\hline & & P: 0.06 & P: 0.001 & \\
\hline
\end{tabular}

Table 4: Comparison of oxidant and antioxidant indices in the diabetic and nondiabetic patients with mild cognitive impairment.

Table 4 indicates a complete description of comparison between the oxidant and antioxidant indexes in the two groups. Regarding the control group, the oxidative indexes including Advanced Glycation End Products (AGEs) and Advanced oxidation protein products (AOPP) decreased significantly ( $\mathrm{p}$ value $<0.001$, df: 48 ). As for the intervention group, the oxidant indexes including AGEs and AOPP, as well as LAP (LipoProtein Lipase) and Lecithin-cholesterol acyltransferase (LCAT) antioxidant indexes decreased significantly ( $\mathrm{p}$ value $<0.001$, df: 48 ).

A significant difference was observed in oxidant/antioxidant indices in both groups before and after study. The difference between AGEs and LPL before and after the study showed a significant difference between the control and intervention groups ( $\mathrm{p}$ value $<0.001$, df: 48). In addition, differences between AOPP and LCAT before and after the study were statistically different in both control and intervention groups ( $\mathrm{p}$-value $=0.02$, df: 48 ; $\mathrm{p}$ value: 0.02 , df: 48). However, the difference between Ferritin Reducing Ability of Plasma and Paraoxonase- 1 before and after the study did not reveal any significant difference between the control and intervention groups ( $\mathrm{p}$ value $=0.98$, df: 48 ; P value: 0.38 , df: 48 ).

\section{Results}

Among 50 subjects in this study, 22 were men and 28 women (each group: 11 men and 14 women). The mean age of the subjects was $72 / 80$ \pm 3.95 (age range: 80- 66 years). Anthropometric properties before study are presented in Table 2.

As shown in Table 3, weight, BMI and systolic and diastolic blood pressures were compared in both groups. There was no correlation between weight and BMI with systolic and diastolic blood pressures before and after study of two groups (P value: n.s). Systolic blood pressure before and after the study indicated a significant difference between the control and intervention groups ( $\mathrm{P}$ value $<0.001$, df: 48).

The subjects included 22 men and 28 women. The mean age subject was $72.87 \pm 3.95$. Regarding the control group, the oxidative indexes including Advanced Glycation End Products (AGEs) and Advanced oxidation protein products (AOPP) decreased significantly ( $\mathrm{p}$ value $<0.001$, df: 48 ). Regarding the intervention group, the oxidant indexes including AGEs and AOPP significantly decreased. In addition, antioxidant indexes such as LAP and Lecithin-cholesterol acyltransferase (LCAT) indexes increased considerably ( $\mathrm{p}$ value $<0.001$, df: 48).

\section{Discussion}

In this study, both control and intervention groups were suffering from Prediabetic State and MCI and were receiving Metformin. Memantine was prescribed for the intervention group. Based on the results, systolic blood pressure decreased significantly before and after the study and the oxidative indexes of AGEs and AOPP decreased significantly in the intervention group. In addition, LPL and LCAT antioxidant indices increased significantly and Hemoglobin subunit alpha 1 significantly decreased in this group. Regarding the control group, oxidative indexes including AGEs and AOPP decreased significantly while no change occurred in other oxidant/antioxidants. However, a reduction of Fasting Blood Sugar occurred in both groups.

Based on strong evidence, there is a disturbance in the balance of oxidant/antioxidant in cognitive dysfunction [17]. Disorder in calcium homeostasis is regarded as one of the mechanisms which occurs at the cellular level in cognitive impairment and causes a collapse of the oxidant/antioxidant balance [4] and mitochondrial calcium content affects its function. Memantine is an antagonist for N-methyl-Daspartate receptor (NMDA) which can attach to NMDA receptor channels and prevents calcium penetration. Thus, it can prevent calcium homeostasis disorder and increase free radicals [21]. Further, in the study of Özdemir, it was found that Memantine can reduce the oxidative stress in ischemic brain injuries [22].

There is a risk of cognitive decline among the people with diabetes type II and prediabetes [9]. Clinical evidences suggest increased reactive oxygen species (ROS) in diabetes, and high levels of intracellular and extracellular glucose lead to oxidative stress [28]. The total oxidant level is high among the people with diabetes type 2 and prediabetes $[29,30]$, and chronic hyperglycemia, due to oxidative stress, has led to changes in mitochondria function and the structure of the central nervous system, which ultimately leads to diabetic cognitive dysfunction [31]. Therefore, the rate of cognitive change in diabetic patients with mild cognitive impairment is higher than that of other people with mild cognitive impairment. Memantine preserves the energy status of the brain during hypoglycemia, which prevents from disrupting calcium homeostasis [21,32]. After cognitive complications, the level of glucose fluctuations decreases among the people with diabetes type II and prediabetes.

\section{Conclusion}

The results of this study revealed that simultaneous administration of Memantine and Metformin in elderly patients with Prediabetes and Mild Cognitive Impairment may decrease oxidant indexes and increase antioxidant indexes, which results in reducing the effects of oxidative stress. 


\section{Limitations of the study}

Based on the results, the simultaneous administration of Memantine and Metformin may influence the studied indexes. Therefore, further research is needed to confirm the prescription of Memantine for those people with diabetes and MIC. Finally, some other studies can be conducted with more subjects.

\section{Acknowledgments}

We would like to thank the subjects in this study and all those who helped us to conduct the present study.

\section{References}

1. Afshar PF, Foroughan M, Vedadhir A, Tabatabaei MG (2017) The effects of place attachment on social well-being in older adults. Educational Gerontology 43: $45-51$

2. Bloom DE, Canning D, Fink G (2010) Implications of population ageing for economic growth. Oxford Rev Econ Pol 26: 583-612.

3. Tabrizi LB, Navab E, Afshar PF, Noghabi AAN, Haghani H (2015) Effect of cognitive-behavioral intervention on burden of family caregivers of patients with alzheimer's disease. Journal of Hayat 21: 94-102.

4. Fillit HM, Rockwood K, Young JB (2016) Brocklehurst's textbook of geriatric medicine and gerontology e-book. 8th ed. Philadelphia: Elsevier Health Sciences, p: 1168

5. Kwon JY, Yang JH, Han JS, Kim DG (2017) Analysis of the retinal nerve fiber layer thickness in alzheimer disease and mild cognitive impairment. Korean $\mathrm{J}$ Ophthalmol 31: 548-556.

6. Seo K, Kim JK, Oh DH, Ryu H, Choi H (2017) Virtual daily living test to screen for mild cognitive impairment using kinematic movement analysis. PloS one 12: e0181883.

7. Wang F, Zhao M, Han Z, Li D, Zhang S, et al. (2017) Association of body mass index with amnestic and non-amnestic mild cognitive impairment risk in elderly. BMC psychiatry 17: 334.

8. Weinstein AM, Barton C, Ross L, Kramer JH, Yaffe K (2009) Treatment practices of mild cognitive impairment in California alzheimer's disease centers. J Am Geriatr Soc 57: 686-690.

9. Hazari MAH, Reddy BR, Uzma N, Kumar BS (2015) Cognitive impairment in type 2 diabetes mellitus. Diabetes Res Clin Pract 3: 19-24.

10. Saedi E, Gheini MR, Faiz F, Arami MA (2016) Diabetes mellitus and cognitive impairments. World journal of diabetes 7: 412 .

11. Yuan XY, Wang XG (2017) Mild cognitive impairment in type 2 diabetes mellitus and related risk factors: A review. Rev Neurosci 28: 715-723.

12. Flöel A, Rujescu D, Kerti L, Grittner U, Witte V (2014) Impact of glucose levels on memory functions and hippocampal microstructure in older adults. Alzheimers Dement 10: 125-126.

13. Lily M, Godwin M (2009) Treating prediabetes with metformin systematic review and meta-analysis. Can Fam Physician 55: 363-339.

14. Bitra V, Rapaka D, Akula A (2015) Prediabetes and alzheimer's disease. Indian J Pharm Sci 77: 511-544.
15. Crane PK, Walker R, Hubbard RA, Li G, Nathan DM, et al. (2013) Glucose Levels and Risk of Dementia. N Engl J Med 369: 540-548.

16. Kawamura T, Umemura T, Hotta N (2012) Cognitive impairment in diabetic patients: Can diabetic control prevent cognitive decline? Journal of Diabetes Investigation 3: 413-423

17. Wojsiat J, Zoltowska KM, Laskowska-Kaszub K, Wojda U (2018) Oxidant/ antioxidant imbalance in alzheimer's disease: Therapeutic and diagnostic prospects. Oxidative medicine and cellular longevity pp: 1-16.

18. Riederer P, Hoyer S (2006) From benefit to damage, glutamate and advanced glycation end products in Alzheimer brain. J Neural Transm 113: 1671-1677.

19. Banzi R, Camaioni P, Tettamanti M, Lucca U (2016) Older patients are still underrepresented in clinical trials of alzheimer's disease. Alzheimers Res Ther 8: 32.

20. Reisberg B, Doody R, Stöffler A, Schmitt F, Ferris S, et al. (2003) Memantine in moderate-to-severe Alzheimer's disease. N Engl J Med 348: 1333-1341.

21. McAllister J, Ghosh S, Berry D, Park M, Sadeghi S, et al. (2008) Effects of memantine on mitochondrial function. Biochem pharmacol 75: 956-964.

22. Özdemir H, Ilhan S, Demir C, Akgün B, Kapan O, et al. (2014) Effects of memantine and clopidogrel alone and in combination on cerebral ischemia in rats (P1. 098). Neurol 82: 98.

23. Halter JB, Ouslander JG, Studenski S, High KP, Asthana S, et al. (2017) Hazzard's geriatric medicine and gerontology. New York: McGraw-Hill Education Medical p: 2520.

24. Flanigan PM, Khosravi MA, Leverenz JB, Tousi B (2018) Color vision impairment differentiates alzheimer dementia from dementia with lewy bodies. J Geriatr Psychiatry Neurol 31: 97-102.

25. König M, Pfarr C, Zweifel P (2014) Mutual altruism: Evidence from alzheimer patients and their spouse caregivers preference measurement in health. Emerald group publishing limited p. 141-160.

26. Olazarán J, Hernández-Tamames JA, Molina E, García-Polo P, Dobato JL, et al. (2013) Clinical and anatomical correlates of gait dysfunction in Alzheimer's disease. J Alzheimers Dis 33: 495-505.

27. Rao N, Chou T, Ventura D, Abramowitz W (2005) Investigation of the pharmacokinetic and pharmacodynamic interactions between memantine and glyburide/metformin in healthy young subjects: a single-center, multiple-dose, open-label study. Clinical Therapeutics 27: 1596-606.

28. Matough FA, Budin SB, Hamid ZA, Alwahaibi N, Mohamed J (2012) The role of oxidative stress and antioxidants in diabetic complications. Sultan Qaboos Univ Med J 12: 5

29. Erdem SS, Toker A, Kayrak M, Çiçekler H, Gönülalan G, et al. (2015) Oxidant and antioxidant parameters in prediabetes and diabetes. Int J Diabetes Dev Ctries 35: 465-470.

30. Saygili E, Aksoy S, Gurler B, Aksoy A, Erel O, et al. (2010) Oxidant/antioxidant status of patients with diabetic and senile cataract. Biotechnol Biotec Eq 24 1648-1652.

31. Zhang Q, Kong M, Li H, Li J, Zhang $\mathrm{H}$, et al. (2017) Effect of alpha lipoic acid on cognitive function and oxidative stress in STZ diabetic rats. Biomed Res 28: 12.

32. Willenborg B, Schmoller A, Caspary J, Melchert U, Scholand-Engler H, et al (2011) Memantine prevents hypoglycemia-induced decrements of the cerebral energy status in healthy subjects. J Clin Endocrinol Metab 96: 384-388. 